\title{
Levantamento e observações naturalísticas da acarofauna (Acari, Arachnida) de seringueiras cultivadas (Hevea spp., Euphorbiaceae) no Brasil
}

\author{
Reinaldo J.F. Feres ${ }^{1}$
}

\begin{abstract}
Survey and naturalistic remarks on the acarological fauna (Acari, Arachnida) from Hevea spp. (Euphorbiaceae) in Brazil. This paper reports twenty eight acarine species belonging to twenty four genera in eleven families, associated with rubber trees, Hevea spp. (Euphorbiaceae), from Brazil. A key to the genera and species is included.

KEY WORDS. Acari, mites, naturalistic remarks, Hevea, rubber tree, Brazil
\end{abstract}

$\mathrm{O}$ estudo da fauna acarina associada às seringueiras se reveste de grande importância, pois a implantação da heveacultura em vários Estados do Brasil, associada a fatores climáticos das novas regiões em que foram introduzidas, tem propiciado a associação de várias espécies de ácaros com essas plantas.

Os trabalhos enfocando a acarofauna de seringueiras no Brasil, restringem-se aos de Silva (1972) e de FleCHTMANN \& ARLEU (1984). Outros trabalhos referem-se à descrição de novas espécies (BAKER 1945; FERES 1992, 1998), ou de novo registro de ácaro nessas plantas (FAZOLIN \& PEREIRA 1989; FLECHTMANN 1989).

O material coletado em parte pelo autor e em parte enviado por profissionais ligados à heveacultura, consistiu em folíolos, peciólulos e pecíolos ("hastes") de dez clones de Hevea brasiliensis e de outras cinco espécies de Hevea, cultivadas em sistema de monocultura nos Estados de Minas Gerais, Mato Grosso, Mato Grosso do Sul e São Paulo. De cada amostra coletada ou recebida para estudos, cerca de 20 folíolos foram examinados e todos os ácaros presentes foram montados em lâminas de microscopia, com a utilização do meio de Hoyer (FLECHTMANN 1975; JEPPSON et al. 1975).

O estudo taxonômico dos exemplares de ácaros associados a esse material revelou 28 espécies pertencentes a 24 gêneros de 11 famílias, além de deutoninfas hipopiais de Histiostomatidae. A nomenclatura adotada para as categorias superiores é aquela proposta por WoLLEY (1987). As observações realizadas no campo e em laboratório, além de dados obtidos junto a produtores e profissionais ligados a heveacultura, permitiram o registro do microhabitat e dos sintomas apresentados pelas novas infestações nessa cultura. Para as espécies de ocorrência mais freqüente nas amostras, pôde-se registrar a ocorrência sazonal. São usadas as seguintes abreviaturas: (F) fêmea, (M) macho.

Uma chave dicotômica para auxiliar na identificação das famílias, gêneros e espécies registradas, foi elaborada e é apresentada.

O material estudado está depositado na coleção de Acari, do Departamento de Zoologia, Universidade Estadual Paulista (UNESP), São José do Rio Preto, São Paulo.

1) Departamento de Zoologia, Universidade Estadual Paulista. Caixa Postal 136, 15051-970 São José do Rio Preto, São Paulo, Brasil. E-mail: reinaldo@zoo.ibilce.unesp.br 
Chave para identificação das ordens, famílias, gêneros e espécies dos ácaros registrados em seringueiras no Brasil

1. Tarso do palpo com apotele; tritosterno presente; estigmas localizados entre as coxas III e IV . . . . . . . . . . 2

- . Tarso do palpo sem apotele; tritosterno e estigmas entre coxas III e IV ausentes . . 4

2. Setas z3 e s6 ausentes; setas R1 e S5 presentes; macrosetas presentes nas pernas Euseius ..... 3

-. Setas z3 e s6 presentes; setas R1 e S5 ausentes; macrosetas das pernas ausentes Typhlodromus annectens

3. Escudo dorsal levemente reticulado; macroseta St IV terminando em ponta recurvada ........................... Euseius citrifolius

- Escudo dorsal liso; macrosetas com pontas espatuladas .........E. concordis

4. Empódio unciforme, situado em um distinto prétarso ou carúncula; unhas verdadeiras ausentes; sistema traqueal ausente; palpos simples; quelíceras quelado-denteadas; abertura genital em forma de $\mathrm{V}$ invertido .......... Astigmata . . . 27

- . Empódio pode estar ausente; se presente, empódio de formas variadas, mas se unciforme então com vários pelos ventrais; sistema traqueal geralmente presente, com estigmas abrindo-se entre as bases das quelíceras ou na base do gnatossoma ou anteriormente no propodossoma; quelíceras estiletiformes, raramente queladas; abertura genital não como acima ............

Actinedida . . . . 5

5. Gnatossoma sem a forma de cápsula; palpos distintos, se não distintos o corpo é vermiforme e o ácaro apresenta dois pares de pernas em todos os estágios, em alguns grupos pode apresentar uma seta terminal da tíbia modificada em esporão que desloca o tarso lateral ou ventralmente (complexo "unha-

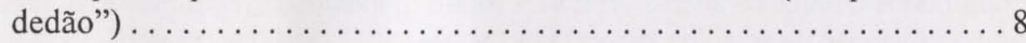

-. Gnatossoma como uma cápsula ovóide; palpos diminutos lateralmente aderentes; quarto par de pernas da fêmea apresenta duas longas setas flageliformes distais; empódio como estrutura membranosa com garra firmemente ligada . . . . . . . . . . . . . . 6

6. Fêmea com prodorso e tergitos $\mathrm{C}, \mathrm{D}, \mathrm{EF}$ fortemente reticulados

. Daidalotarsonemus

- . Fêmea com prodorso e tergitos C,D, EF lisos

7. Tarso IV do macho terminando numa estrutura em forma de botão arredondado . Polyphagotarsonemus latus

- . Tarso IV do macho terminando em um robusto esporão ......... Tarsonemus

8. Corpo vermiforme/fusiforme; com dois pares de pernas . . Eriophyidae . . . .99

- . Corpo ovalado/arredondado, com mais de dois pares de pernas . . . . . . . 11

9. Escudo dorsal sem setas, com margens anterior e laterais reticụladas; empódio

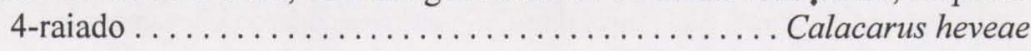


- . Escudo dorsal com setas inseridas em tubérculos bem desenvolvidos, com eixo basal longitudinal e dirigidas para cima; margens do escudo não reticuladas; empódio 4 ou 5 -raiado .......................... 10

10. Tubérculo dorsal localizado junto à margem posterior do escudo dorsal; empódio 5-raiado; anéis do opistossoma uniformemente arqueados .......... Shevtchenkella petiolula

- . Tubérculos dorsais localizados anteriormente à margem posterior do escudo dorsal; empódio 4-raiado; anéis do opistossoma com largo sulco dorso-mediano, flanqueado de cada lado por lobos arredondados com microtubérculos bem desenvolvidos ................. Phyllocoptruta seringueirae

11. Palpo com seta dorsal da tíbia modificada em esporão, que desloca o tarso lateral

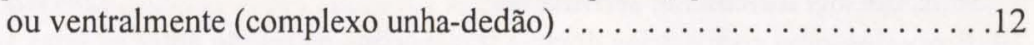

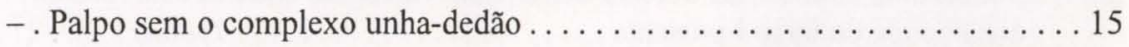

12. Quelíceras como estiletes longos e recurvados, implantados em um estilóforo; tarsos I e II com setas dúplices ou associadas; região ao redor da abertura genital da fêmea pregueada . . . . . . . . . . . Tetranychidae . . 23

- . Quelíceras curtas e afiladas, não implantadas em estilóforo; tarsos sem setas dúplices ou associadas; região ao redor da abertura genital da fêmea não pregueada .................................. 13

13. Bases das quelíceras fundidas com o restante do gnatossoma; tíbia do palpo com seta modificada robusta e em forma de foice e tarso do palpo com duas setas pectinadas; segmentos do palpo fortemente mais robustos que os das pernas . Cheyletidae ..... Hemicheyletia wellsi

-. Bases das quelíceras fundidas ao longo de sua linha mediana; tíbia do palpo com a seta modificada sem a forma de foice e tarso do palpo sem as setas pectinadas; segmentos do palpo de espessura semelhante a das pernas .... ............................ Stigmaeidae . . 14

14. Escudo central do histerossoma da fêmea com cinco pares de setas e do macho

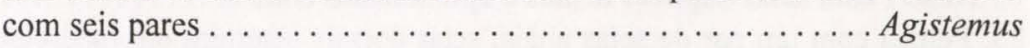

-. Escudo central do histerossoma da fêmea e do macho com quatro pares de setas

Zetzellia

15. Bases das quelíceras não fundidas; com dois pares de longas setas sensoriais no dorso do propodossoma ............. Cunaxidae ..... Pseudobonzia

- . Bases das quelíceras fundidas podendo formar um estilóforo; com somente um par de setas sensoriais no dorso do propodossoma ou sem essas setas . . 16

16. Corpo achatado dorso-ventralmente; dígitos móveis das quelíceras modificados em estiletes longos e recurvados; setas sensoriais propodossomais ausentes $\ldots \ldots \ldots \ldots \ldots \ldots \ldots \ldots \ldots \ldots$ Tenuipalpidae . . . 17

- . Corpo não achatado; dígitos móveis das quelíceras modificados em estiletes curtos e retos; com um par de setas sensoriais no dorso do propodossoma...

Tydeidae .... 18 
17. Palpo com três segmentos; podossoma tipicamente mais largo que o opistossoma; penúltimo par de setas dorsolaterais (L5 ou h2) flageliforme ......... .................................. Tenuipalpus heveae

- . Palpo com quatro segmentos; podossoma não distintamente mais largo que o opistossoma; penúltimo par de setas dorsolaterais (L5 ou h2) de comprimento normal .

. Brevipalpus phoenicis

18. Estrias dorsais do tegumento formando padrão reticulado na totalidade ou parte do idiossoma .......................... Lorryia . . . 19

- . Estrias dorsais do tegumento não formam padrão reticulado .

19. Setas dorsais lanceoladas e lisas, com metade apical tipicamente expandida e recurvada ............................ Lorryia formosa

- . Setas dorsais fortemente serrilhadas . . Lorryia boycei

20. Propodossoma com estrias dorsais transversais na região entre as setas sensoriais; apotele do tarso I presente ................. Tydeus . . . 21

-. Propodossoma com estrias dorsais longitudinais entre as setas sensoriais; apotele

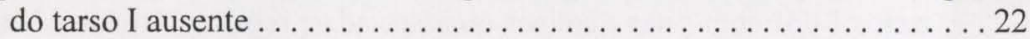

21. Estrias da região entre as setas D1 e D2 formando um padrão em forma de V.

- . Estria da região entre as setas D1 e D2 sem esse padrão ........... Tydeus

22. Seta anal ausente, fêmures III e IV apresentando, cada um, uma proeminente seta bifurcada .......................... Parapronematus

- . Com um simples par de setas anais; setas dos fêmures III e IV normais . . .....

Pronematus

23. Tarso I sem setas dúplices mas com setas associadas; empódio ausente; dois pares de setas paranais . .................. Eutetranychus banksi

- . Tarso I com setas dúplices; empódio unciforme ou dividido distalmente; com um par de setas paranais . ......................... 24

24. Tarso I com setas dúplices bem separadas, dividindo o segmento em terços aproximadamente iguais ................ Tetranychus mexicanus

- . Tarso I com setas dúplices distais e aproximadas entre si . . . . . . . . 25

25. Fêmea com um par de setas anais; setas dorsais inseridas em tubérculos bem desenvolvidos . .................... Atrichoproctus uncinatus

- . Fêmea com dois pares de setas anais; setas dorsais inseridas diretamente no tegumento ......................... Oligonychus ....26

26. Tíbia I da fêmea com sete setas táteis (e um sensorial); edéago dirigido ventralmente terminando em ponta .............. Oligonychus coffeae

- Tíbia I da fêmea com nove setas táteis (e um sensorial); edéago dirigido dorsalmente, com cabeça sigmóide ................. gossypii

27. Empódio unciforme unido à extremidade do tarso por um par de escleritos circundados por um curto pretarso

Acaridae ..... Tyrophagus putrescentiae

-. Empódio unciforme inserido na extremidade de um pretarso unido à extremidade do tarso por apenas um delicado tendão . 


\section{Phytoseiidae Berlese, 1916 Euseius citrifolius Denmark \& Muma, 1970}

Euseius citrifolius Denmark \& Muma, 1970: 222. - Moraes \& McMurtry, 1983: 138. -Feres \& Moraes, 1998: 125.

Material examinado. 6F, 13-VI-1990, H. brasiliensis, Ibitinga, São Paulo; 1F, 06-VIII-1991, H. brasiliensis - clone PB 235, Frutal, Minas Gerais; 2F e 1M, 7-VI e 1F, 14-VI-1991, H. brasiliensis, Macaubal, São Paulo; 1F, 27-II-1996, H. brasiliensis, Barretos, São Paulo; 6F e 1M, 02-X-1997, H. brasiliensis - clones GT 1, PB 235, PB 260; 1F, 30-X-1997, H. brasiliensis - clone PB 260; 1F, 13-I e 1F e 1M, 30-I-1998, H. brasiliensis - clone PB 235, Rondonópolis, Mato Grosso.

Distribuição. Brasil, Bahia, Ceará, Paraíba, Pernambuco, São Paulo; Paraguai, Peru (MORAES et al. 1986); Colômbia e Nicarágua (MORAES et al. 1991).

Observações. Esta espécie de fitoseídeo ocorre sobre diversas espécies de plantas. Na região noroeste do Estado de São Paulo é a mais freqüente e abundante (FERES \& MORAES 1998). Predador de outros ácaros, principalmente daqueles pertencentes à família Tetranychidae.

\section{Euseius concordis (Chant, 1959)}

Typhlodromus (Amblyseius) concordis Chant, 1959: 69-70.

Amblyseius (Iphiseius) concordis; Muma, 1961: 288.

Amblyseius concordis; Chant \& Baker, 1965: 22. - Moraes \& McMurtry, 1983: 138.

Euseius concordis; Denmark \& Muma, 1973: 264. - Moraes \& Oliveira, 1982: 317. - Moraes \& McMurtry, 1983: 138. - Feres \& Moraes, 1998: 127.

Euseius flechtmanni Denmark \& Muma, 1970: 223. - Denmark \& Muma, 1973: 261 (sinônimo, de acordo com Moraes et al., 1982: 18).

Material examinado. 3F e 3M, 02-X-1997, H. brasiliensis - clones PB 217 e PB 260; 4F e 1M, 30-X-1997, H. brasiliensis - clones GT 1, PB 217 e PB 260; 1M, 30-I e 1F, 14-V-1998, H. brasiliensis - clone PB 217, Rondonópolis, Mato Grosso.

Distribuição. Argentina; Brasil (Bahia, Ceará, Paraíba, Pernambuco, São Paulo); Colômbia; Costa Rica; El-Salvador; Guatemala; Nicarágua; Portugal; Trinidad-Tobago; Iugoslávia (MORAES et al. 1986).

\section{Typhlodromus annectens DeLeon, 1958}

Typhlodromus annectens DeLeon, 1958: 75. - Chant \& Yoshida-Shaul, 1984: 1868. - Moraes \& McMurtry, 1983: 142. - Moraes \& Mesa, 1988: 82. - Moraes et al., 1991: 134. - Feres \& Moraes, 1998: 129.

Galendromus annectens Muma, 1961: 298. - Muma, 1963: 20; Muma et al., 1970: 135. - Denmark \& Muma, 1973: 274. - Farias et al., 1981: 316. - Moraes et al., 1982: 21.

Material examinado. 2F e 1M, 02-X-1997, H. brasiliensis - clone PB 260, Rondonópolis, Mato Grosso.

Distribuição. Brasil (Pernambuco, São Paulo; Canadá); Colômbia; E.U.A.; Galápagos; Honduras; Jamaica; México; Porto Rico (MORAES et al. 1986).

\section{Cunaxidae Thor, 1902 \\ Pseudobonzia sp.}

Material examinado. 1F, 14-V-1998, H. brasiliensis - clone GT 1, Rondonópolis, Mato Grosso. 
Observações. Ácaros da família Cunaxidae são predadores de cochonilhas e de outros pequenos artrópodes (SMILEY 1975).

\section{Cheyletidae Leach, 1815}

\section{Hemicheyletia wellsi (Baker, 1949a)}

Cheyletia; wellsi Baker, 1949a: 300-301.

Paracheyletia; wellsi Volgin, 1955: 152. - Muma, 1964: 245-246.

Hemicheyletia; wellsi Summers \& Price, 1970: 18.

Material examinado. 1F, IV-1996, H. brasiliensis, Reginópolis, São Paulo.

Distribuição. África (RoDRIGUES 1968); Brasil (São Paulo) (FERES \& FLECHTMANN 1995); E.U.A. (MUMA 1964).

Observações. A maioria das espécies é predadora de grande variedade de pequenos artrópodes, como ácaros e Collembola. Algumas espécies ocorrem sobre a folhagem das plantas onde se alimentam de ácaros fitófagos e cochonilhas (FLECHTMANN 1975). H. wellsi tem sido coletada com facilidade em folhas e frutos de citros atacados por Phyllocoptruta oleivora (Ashmead), o ácaro da falsa ferrugem (Chiavegato 1980).

\section{Stigmaeidae Oudemans, 1931 Agistemus sp.}

Material examinado. 1M, 13-VI-1990, H. brasiliensis, Ibitinga, São Paulo; 5F e 1M, I-1996, H. brasiliensis, Barretos, São Paulo; 2F, 14-VII-1997, H. pauciflora; 2F, 04-VIII-1997, H. benthamiana e $H$. pauciflora; 2F, 02-X e 1F, 25-XI-1997, H. brasiliensis - clone PB 260; 1M, 13-I-1998, H. brasiliensis - clone PB 235; 3F, 30-I-1998, H. brasiliensis - clone RRIM 600, 1M, 22-IV-1998 e 1F e 2 M, 14-V-1998, H. brasiliensis - clones GT1, PB 217, PB 235, RRIM 600, Rondonópolis, Mato Grosso.

Observações. Espécies desse gênero são geralmente conhecidas como predadores ativos de ovos de tetraniquídeos (MCMURTRY et al. 1970; OOMEN 1982; INOUE \& TANAKA 1983 apud EHARA 1993). Ácaro referido como predador de Tenuipalpus spp. (FLECHTMANN 1975). Muito comum, ocorrendo sobre grande número de plantas. Sua coloração varia do brancacento ao vermelho amarronzado.

\section{Zetzellia sp.}

Material examinado. 1F, 13-VI-1990, H. brasiliensis, Ibitinga, São Paulo; 2F, 25-XI-1997, H. brasiliensis - clone PB 235, Rondonópolis, Mato Grosso.

Distribuição. Gênero com espécies de ampla distribuição geográfica (WOOD 1967).

Observações. É conhecido o hábito alimentar de Z. mali (Ewing, 1921), que se alimenta de várias espécies de ácaros tetraniquídeos na América do Norte, Europa e Israel (JEPPSON et al. 1975).

\section{Tydeidae Kramer, 1877 \\ Lorryia formosa Cooreman, 1958}

Lorryia formosa Cooreman, 1958: 6-10. - Baker, 1968: 995-996.

Material examinado. 11F, 13-VI-1990, H. brasiliensis, Ibitinga, São Paulo; 
1F, 30-I-1998, H. brasiliensis - clone PB 235; 2F, 14-V-1998, H. brasiliensis clone RRIM 600, Rondonópolis, Mato Grosso.

Distribuição. Argentina, Brasil, Equador, Espanha, França, Marrocos, México, Uruguai (BAKER 1968), Paraguai (FLECHTMANN 1973).

Observações. Ácaros de coloração verde-amarelada. Espécie não identificada deste gênero foi registrada em seringueiras do Município de Rio Claro, São Paulo, por FLECHTMANN \& ARLEU (1984). Ocorrem praticamente ao longo do ano, sobre um grande número de plantas, sem causar dano aparente às folhas.

\section{Lorryia boycei (Baker, 1944)}

Retetydeus boycei Baker, 1944: 78.

Lorryia boycei; Baker, 1968: 1004.

Material examinado. 1F, IV-1996, H. brasiliensis, Reginópolis, São Paulo.

Distribuição. E.U.A., México (BAKER 1968).

Observações. Espécie registrada por BAKER (1968) sobre musgo e sob o súber de camélia.

\section{Tydeus kenyensis Baker, 1970}

Tydeus kenyensis Baker, 1970: 165

Material examinado. 1F, 07-VI-1991, H. brasiliensis, Macaubal, São Paulo; 2F, 14-V-1998, H. brasiliensis - clone PB 217, Rondonópolis, Mato Grosso.

Distribuição. Quênia (BAKER 1970). 1970).

Observações. Espécie descrita originalmente de folhas de cafeeiro (BAKER

\section{Tydeus sp.}

Material examinado. 2F, 10-II-1997, H. brasiliensis - clone RRIM 600, Monte Aprazivel, São Paulo.

Observações. Pouco se conhece a respeito da biologia das espécies pertencentes a este gênero. Podem ser encontrados em grande número, sobre folhas de plantas diversas, no húmus e fragmentos de plantas, e sobre produtos alimentícios armazenados (BAKER 1970).

\section{Pronematus sp.}

Material examinado. 1M e 1F, 13-VI-1990, H. brasiliensis, Ibitinga, São Paulo; 1F, 14-VI-1991, H. brasiliensis, Macaubal, São Paulo; 1F, IV-1996, Reginópolis, São Paulo; 2F e 1M, 30-IV-1996, Pindorama, São Paulo; 1F e 1M, 25-VIII-1997, H. benthamiana, Rondonópolis, Mato Grosso.

Observações. São diminutos, translúcidos e levemente brancacentos e de movimentos rápidos. São referidos como predadores de eriofiídeos (BAKER \& WHARTON 1952).

\section{Parapronematus sp.}

Material examinado. 1F, 14-V-1991, H. brasiliensis, José Bonifácio, São Paulo; 1M, 07-VI-1991, H. brasiliensis, Macaubal, São Paulo; 1M, I-1996, H. brasiliensis, Barretos, São Paulo. 
Observações. Semelhantes a Pronematus sp. na forma e coloração, diferem entretanto por apresentarem setas bífidas sobre os fêmures III e IV.

\section{Tarsonemidae Kramer, 1877 \\ Polyphagotarsonemus latus (Banks, 1904).}

Tarsonemus latus Banks, 1904: 1553.

Hemitarsonemus latus; Ewing, 1939: 54.

Neotarsonemus latus; Smiley, 1967: 137.

Polyphagotarsonemius latus; Beer \& Nucifora, 1965: 38-42.

Material examinado. 6F e 1M, 06-VIII-1991, H. brasiliensis - clone PB 235, Frutal, Minas Gerais.

Distribuição. Ampla distribuição mundial, em países de regiões tropicais e temperadas quentes (LINDQUIST 1986).

Observações. Espécie conhecida como ácaro branco ou tropical e praga de diversas culturas. Já registrada sobre seringueiras (SILVA 1972; JEPPSON et al. 1975); no Brasil foi registrada pela primeira vez em seringueiras do Espirito Santo (FLECHTMANN \& ARLEU 1984). Seu ataque nessas plantas restringe-se aos brotos novos e gemas. Prefere clima quente e úmido (FLECHTMANN 1975).

\section{Daidalotarsonemus sp.}

Material examinado. 1F, 22-IV e 1M, 14-V-1998, H. brasiliensis-clone GT 1, Rondonópolis, Mato Grosso.

Distribuição. Ácaros pertencentes a este gênero ocorrem em regiões temperadas e tropicais em várias regiões do mundo (LINDQUIST 1986).

Observações. Possuem hábitos alimentares não bem definidos, mas provavelmente alimentam-se de algas e fungos (LINDQUIST 1986).

\section{Tarsonemus sp.}

Material examinado. 1F, 06-VI-1997, H. brasiliensis - clone PB 260; 4F, 22-IV-1998, H. brasiliensis - clone GT 1, Rondonópolis, Mato Grosso.

Distribuição. As espécies pertencentes a este gênero são geralmente cosmopolitas (LINDQUIST 1986).

Observações. São primariamente micófagos (LINDQUIST 1986).

Tenuipalpidae Berlese, 1913 Brevipalpus phoenicis (Geijskes, 1939)

Tenuipalpus phoenicis Geijskes, 1939: 23.

Brevipalpus phoenicis; Sayed, 1946. - Pritchard \& Baker, 1958: 233, - DeLeon, 1961: 48. - Gonzalez, 1975: 82. - Baker et al., 1975: 18. - Meyer, 1979: 87. - Baker \& Tuttle, 1987: 98-99.

Brevipalpus yothersi Baker, 1949b: 374.

Brevipalpus mcbridei Baker, 1949b: 374.

Brevipalpus papayensis Baker, 1949b: 379.

Material examinado. 17F, 13-VI-1990, H. brasiliensis, Ibitinga, São Paulo; 5F, 14-V-1991, H. brasiliensis, José Bonifácio, São Paulo; 6F, 07-VI e 1F, 14-VI- 
1991, H. brasiliensis, Macaubal, São Paulo; 1F, IV-1996, H. brasiliensis, Reginópolis, São Paulo.

Distribuição. Espécie de ampla distribuição geográfica, ocorrendo sobre grande número de espécies de plantas hospedeiras. África (MEYER \& RODRIGUES 1965; Rodrigues 1968; Meyer 1979); Brasil (Alagoas, Bahia, Ceará, Minas Gerais, Paraná, Pernambuco, Rio de Janeiro, São Paulo) (FlechtMAnN 1976); Colômbia (Zuluaga \& SALDARRIAGa 1970); E.U.A. (BAKER \& TUTTLE 1987); Porto Rico (COMRoY 1958).

Observações. Referido como "ácaro da leprose dos citros", pois é o vetor da virose que causa essa patogenia nos citros (CHIAVEGATO 1980). Já foi registrado em seringueiras de Itabuna, Bahia e Rio Claro, São Paulo (FLECHTMANN \& ARLEU 1984).

\section{Tenuipalpus heveae Baker, 1945}

Tenuipalpus heveae Baker, 1945: 36. - Baker \& Pritchard, 1953: 320.

Material examinado. 30F, I-1996, H. brasiliensis, Barretos, São Paulo; 1F, 30-IV-1996, H. brasiliensis, Pindorama, São Paulo; 8F e 2M, 04-VIII e 1F, 25-VIII-1997, H. viridis; 17F, 30-X-1997, H. brasiliensis - clones PB 260 e RRIM 600; 4F, 25-XI-1997, H. brasiliensis - clones GT 1, PB 217, PB 235, PB 260; 15F, 13-I, H. brasiliensis - clones GT 1, PB 217, PB 235, PB 260, RRIM 600; 4F, 30-I, H. brasiliensis - clones PB 235, PB 260 e RRIM 600; 3F, 22-IV, H. brasiliensis clones GT 1, PB 235; 2F, 14-V, H. brasiliensis - clones PB 217, PB 260; 2F, 31-VII-1998, H. brasiliensis - clone GT 1, Rondonópolis, Mato Grosso.

Distribuição. Brasil, Amazonas (FlechtMANN \& ARLEU 1984), Pará (BAKER 1945).

Observações. Ácaro plano, conhecido no Estado do Pará como ácaro vermelho da seringueira (FLECHTMANN 1979). Ocorrem preponderantemente na face inferior das folhas medianas e mais velhas das seringueiras. Quando em grande infestação, também são encontrados na face superior. As maiores infestações de ácaros dessa espécie têm ocorrido, principalmente durante o mês de fevereiro, em seringais de Barretos, São Paulo.

\section{Tetranychidae Donnadieu, 1875 Eutetranychus banksi (McGregor, 1914)}

Tetranychus banksi McGregor, 1914: 358.

Anychus banksi; McGregor, 1919: 644.

Eutetranychus banksi; Mcgregor, 1950: 268. - Pritchard \& Baker, 1955: 115. - Flechtmann \& Baker, 1970: 156. - Flechtmann \& Baker, 1975: 112.

Material examinado. 1F, 9-VI-1988; 4F e 3M, 18-VIII-1989, Bálsamo, São Paulo; 5F e 3M, 13-VI-1990, Ibitinga, São Paulo; 1F, 07-VI-1991, Macaubal, São Paulo, Hevea brasiliensis; 2F, 06-VIII-1991, Frutal, Minas Gerais, H. brasiliensis - clone PB 235; 1M, 30-IV-1996, H. brasiliensis Pindorama, São Paulo.

Distribuição. África do Sul, Argentina, Egito, E.U.A., Índia, Itália, México, Palestina, Peru (PRITCHARD \& BAKER 1955), Brasil (BondAR 1928; SILVA 1972; FleChTMANN \& ABREU 1973), Porto Rico(COMROY 1958), Maurício (Moutia 1958), Costa Rica, El-Salvador, Nicarágua, Venezuela (BAKER \& PRITCHARD 1962). 
Observações. Nos Estados Unidos da América do Norte esta espécie é considerada séria praga dos citros. No Brasil ocorre sobre grande número de plantas, sem que se tenha registrado danos de importância.

\section{Oligonychus gossypii (Zacher, 1920)}

Paratetranychus gossypii Zacher, 1921: 183.

Oligonychus gossypii; Pritchard \& Baker, 1955: 359. - Baker \& Pritchard, 1960: 508. - Meyer, 1974: 263. - Meyer, 1987: 152.

Material examinado. 1F e 1M, 13-VI-1990, H. brasiliensis, Ibitinga, São Paulo; 2F e 4M, I-1996, H. brasiliensis, Barretos, São Paulo; 1F e 1M, 14-VII-1997, Rondonópolis, Mato Grosso, H. rigidifolia; $1 \mathrm{~F}, 04-\mathrm{VIII}-1997, H$. viridis; $1 \mathrm{~F}$ e $1 \mathrm{M}$, 25-VIII-1997, $H$. viridis e $H$. brasiliensis - clone GT 1; 22F e 6M, 02-X-1997, H. brasiliensis - clones BPM 24 e GT 1; 4F e 10M, 30-X-1997, H. brasiliensis -clones GT 1, PB 217, PB 235, PB 260; 4F e 3M, 25-XI-1997, H. brasiliensis - clones PB 217, PB 235 e PB 260; 1F e 1M, 13-I-1998, H. brasiliensis - clone PB 235; 1F e 1M, 30-I-1998, H. brasiliensis - clones PB 260 e RRIM 600; 1F, 22-IV e 3F, 14-V-1998, H. brasiliensis - clone PB 235; 1F, 31-VII-1998, H. brasiliensis -clone GT 1, Rondonópolis, Mato Grosso.

Distribuição. África (MEYER 1987); Américas Central e do Sul (QuiRÓsGONZALES 1981 apud FLECHTMANN 1989).

Observações. Registrada em vários países da África e nas Américas Central e do Sul, em várias espécies de plantas. Registrada pela primeira vez em seringueiras cultivadas no Brasil em 1986 e 1988, nos estados do Mato Grosso, Acre e Amazonas, causando desfolhamentos (FAZOLIN \& PEREIRA 1989; FleCHTMANN 1989). Habitam a superfície inferior das folhas, onde produzem teias.

\section{Oligonychus coffeae (Nietner, 1861)}

Acarus coffeae Nietner, 1861.

Oligonychus coffeae; Pritchard \& Baker, 1955: 315. - Baker \& Pritchard, 1960: 505-506. - Meyer \& Rodrigues 1965: 12-13. -Rodrigues, 1968: 220. - Gutierrez, 1968: 446-447. - Meyer, 1974: 251-252. - Meyer, 1987: 146-147.

Oligonychus merwei Tucker, 1926: 6.

Paratetranychus terminalis Sayed, 1946: 94.

Material examinado. 3F e 2M, 04-VIII-1997, H. guianensis e H. viridis, Rondonópolis, Mato Grosso.

Distribuição. Espécie de ampla distribuição geográfica e já assinalada em seringueiras em outras partes do mundo (JEPPSON et al. 1975). Brasil, Espírito Santo (FLECHTMANN \& ARLEU 1984).

Observações. Espécie de coloração vermelha, registrada pela primeira vez no Brasil, atacando seringueiras no Estado do Espírito Santo e considerada em muitos países como a mais séria praga do chazeiro (FLECHTMANN \& ARLEU 1984).

\section{Atrichoproctus uncinatus Flechtmann, 1967}

Atrichoproctus uncinatus Flechtmann, 1967: 39 apud Flechtmann \& Baker, 1970: 157. - Flechtmann \& Baker, 1975: 116.

Material examinado. 1F, 04-VIII-1997, H. benthamiana, Rondonópolis, Mato Grosso. 
Distribuição. Brasil, São Paulo (FLECHTMANN \& BAKER 1970).

Observações. Espécie de coloração verde, que ocorre preponderantemente na face superior das folhas.

\section{Tetranychus mexicanus (McGregor, 1950)}

Septanychus mexicanus McGregor, 1950: 323.

Tetranychus mexicanus; Pritchard \& Baker, 1955: 411-412. -Flechtmann \& Baker, 1970: 162. Flechtmann \& Baker, 1975: 120.

Material examinado. 1M, 14-VII-1997, H. pauciflora; 2F e 3M, 04-VIII e $1 \mathrm{~F}$ e $1 \mathrm{M}, 25-\mathrm{VIII}-1997, H$. brasiliensis - clone GT 1, $H$. benthamiana e $H$. pauciflora; 25F e 4M, 02-X-1997, H. brasiliensis - clones BPM 24, GT 1, PB 217, PB 235, PB 260; 18F e 4M, 30-X-1997, H. brasiliensis - clones PB 217, PB 235 e PB 260; 8F e 1M, 25-XI-1997, $H$. brasiliensis - clone PB 235; 1F, 13-I-1998, $H$. brasiliensis - clone PB 235; $1 \mathrm{~F}$ e 1M, 30-I-1998, H. brasiliensis -clones PB 235 e PB 260; 4F e 1M, 22-IV-1998, H. brasiliensis -clones PB 217 e PB 235; 2F e 1M, 14-V-1998, H. brasiliensis - clone PB 235, Rondonópolis, Mato Grosso.

Distribuição. Espécie de ampla distribuição geográfica, ocorrendo em grande número de plantas hospedeiras. Argentina, E.U.A., México (PRITCHARD \& BAKER 1955), Brasil (Bahia, Minas Gerais, Pernambuco, São Paulo) (FLECHTMANN \& BAKER 1970; PASCHOAL 1971); Paraguai (ARANDA \& FLECHTMANN 1971).

Observações. As fêmeas dessa espécie apresentam coloração variável do verde ao vermelho; machos geralmente verde-amarelados ao vermelho. Ocorrem na face inferior das folhas onde produzem apreciável quantidade de teias.

\section{Eriophyidae Nalepa, 1898 \\ Calacarus heveae Feres, 1992}

Calacarus heveae Feres, 1992: 61-65

Material examinado. 41F e 3M, 25-IV e $25 \mathrm{~F}$ e $4 \mathrm{M}, 12$-VI- 1991, $H$. brasiliensis - clones IAN 717, IAN 873, PR 107 e RRIM 600, Planalto, São Paulo; 17F, 15-V, 5F, 07-VI, 13F e 1M, 12-VI e 9F, 21-VI-1991, H. brasiliensis, Macaubal, São Paulo; 20F, 14-V-1991, H. brasiliensis, José Bonifácio, São Paulo; 3F, VII1991, H. brasiliensis - clone PB 235, Frutal, Minas Gerais; 2F, IV-1998, $H$. brasiliensis, Américo de Campos, Mato Grosso; 21F, 18-X-1993, H. brasiliensis, Selvíria, Mato Grosso do Sul; 3F, I-1996, H. brasiliensis, Barretos, São Paulo; 2F, IV-1996, H. brasiliensis, Reginópolis, São Paulo; 17F, 30-IV-1996, H. brasiliensis, Pindorama, São Paulo; 26F, 10-II-1997, H. brasiliensis - clone RRIM 600, Monte Aprazível, São Paulo; 32F e 1M, 06-VI-1997, H. brasiliensis - clone PB 260; 5F, 14-VII-1997, H. brasiliensis - clones PB 235 e PB 260; 32F, 25-XI-1997, $H$. brasiliensis - clones PB 217, PB 260 e RRIM 600; 6F, 13-I, H. brasiliensis - clone GT 1; 9F, 30-I, H. brasiliensis - clone PB 235; 44F e 2M, 22-IV, H. brasiliensis clones FX 3864, PB 217, PB 260, RRIM 600; 34F, 14-V-1998, H. brasiliensis clones GT 1, PB 217, PB 260, Rondonópolis, Mato Grosso.

Distribuição. Brasil (Mato Grosso, Mato Grosso do Sul, Minas Gerais e São Paulo). Espécie descrita da região noroeste do estado de São Paulo (FERES 1992) e agora também registrada em seringais dos Estados de Mato Grosso, Mato Grosso do Sul e Minas Gerais. 
Observações. $\mathrm{O}$ ataque de $C$. heveae ocorre na face superior das folhas de $H$. brasiliensis, durante os meses de outubro a junho, mais comumente nas folhas das regiões sombreadas das plantas. As folhas atacadas adquirem coloração opaca, perdendo seu brilho original, tornando-se amareladas, bronzeadas e caindo com facilidade, no período de abril a maio, portanto antes do período de maior produtividade de látex (maio-junho) na região noroeste do Estado de São Paulo. Este aspecto pode ser confundido com o processo natural de senescência das folhas, que corresponde ao período de julho a agosto. No entanto, neste processo as folhas adquirem coloração avermelhada antes da queda, sem que ocorra queda subseqüente ao rebrotamento. $\mathrm{O}$ ataque intenso desta espécie de ácaro eriofí́deo causa severo desfolhamento em diversos clones de Hevea brasiliensis (AC 53, BPM 24, GT 1, IAN 717, IAN 873, PB 217, PB 235, PB 260, PR 107, RRIM 600) e ocasiona perdas de até $30 \%$ na produção de látex, segundo informações de alguns produtores. Estes também tem notado que os clones IAN 873, PB 235 e 260 são os mais afetados e os clones GT 1 e RRIM 600 os mais resistentes aos efeitos do ataque de $C$. heveae.

\section{Shevtchenkella petiolula Feres, 1998}

Shevtchenkella petiolula Feres, 1998: 69-71.

Material examinado. 18F e 6M, 23-IX e 40F e 19M, 08-X-1993, H. brasiliensis, Buritama, São Paulo; 8F e 1M, 08-X e 5F e 5M, 18-X-1993, H. brasiliensis, Selvíria, Mato Grosso do Sul; 4F e 4M, 25-IX e 31F e 1M, XI-1994, H. brasiliensis, Buritama, São Paulo; 1M, IV-1996, H. brasiliensis, Reginópolis, São Paulo; 1F e 1M, 02-X-1997, H. brasiliensis - clone PB 260, Rondonópolis, Mato Grosso.

Distribuição. Brasil- Mato Grosso, Mato Grosso do Sul e São Paulo. Espécie descrita da região noroeste do Estado de São Paulo e Mato Grosso do Sul (FERES 1998) e agora também registrada no Estado de Mato Grosso.

Observações. Shevtchenkella Bagdalarian, 1968 é um gênero próximo de Tegonotus, entretanto, segundo BAGDASARIAN (1978), Shevtchenkella não é homogêneo em relação a localização ("topografia") dos tubérculos do escudo dorsal e pela direção da seta dorsal neles inserida. Portanto é possível, depois do acúmulo de material comparativo, que este gênero venha a ser dividido em subgêneros.

Trata-se de eriofíídeo de coloração alaranjada, coletado preponderantemente em pecíolos e peciólulos das folhas de seringueiras, em baixa abundância, durante os meses de setembro a novembro.

\section{Phyllocoptruta seringueirae Feres, 1998}

Phyllocoptruta seringueirae Feres, 1998: 71-73.

Material examinado. 1F, 25-IX-1994, H. brasiliensis, Buritama, São Paulo; 36F e 7M, IV-1996, H. brasiliensis, Reginópolis, São Paulo; 20F e 4M, 10-II e 18F e 5M, 19-II-1997, H. brasiliensis - clone RRIM 600, Monte Aprazivel, São Paulo; 159F e 11M, 27-VI-1997; 6F, 14-VII-1997, H. brasiliensis -clones PB 260 e RRIM 600; 4F, 02-X-1997, H. brasiliensis - clones GT 1 e PB 217, Rondonópolis, Mato Grosso.

Distribuição. Brasil (Mato Grosso e São Paulo) (FERES 1998). 
Observações. Esta espécie de eriofiídeo, apresenta como característica típica em relação às demais espécies de Phyllocoptruta Keifer, 1938, os primeiro e segundo anéis dorsais do opistossoma formando uma área irregular atrás do escudo dorsal. Trata-se de espécie de coloração alaranjada, ocorrendo na face inferior das folhas, no período de fevereiro a junho. Essa espécie e $C$. heveae são sincronotópicas, o que dificulta a observação de possíveis danos causados por essa espécie no campo.

\section{Acaridae Ewing \& Nesbitt 1954 Tyrophagus putrescentiae (Schrank, 1781)}

Acarus putrescentiae Schrank, 1781: 521.

Tyrophagus putrescentiae; Oudemans, 1924: 250.

Material examinado. 9F, XI-1994, H. brasiliensis, Buritama, São Paulo; 1F, I-1996, H. brasiliensis, Barretos, São Paulo; 3F e 2M, IV-1996, H. brasiliensis, Reginópolis, São Paulo; 1F, 30-IV-1996, H. brasiliensis, Pindorama, São Paulo; 1F, 30-I-1998, H. brasiliensis - clone PB 235, Rondonópolis, Mato Grosso.

Distribuição. Cosmopolita.

Observações. Espécie registrada como praga de meios de cultura e dietas de insetos em laboratórios e produtos alimentícios armazenados (FLECHTMANN 1986).

\section{Winterschmidtiidae Oudemans, 1923 Oulenzia sp.}

Material examinado. 5M, 13-VI-1990, H. brasiliensis, Ibitinga, São Paulo; 1F, 13-I-1998, H. brasiliensis - clone GT 1; 3F, 30-I-1998, H. brasiliensis - clone PB 235, Rondonópolis, Mato Grosso.

Observações. Ácaros de coloração brancacenta brilhante. $O$. arboricola (Oudemans) foi descrita sobre folhas de Hevea em Sumatra; espécimes também foram coletados sobre juta, na Índia. Relatados como sendo de hábito herbívoro (BAKER \& WHARTON 1952).

\section{Histiostomatidae Hughs, 1976}

Material examinado. XI-1994, H. brasiliensis, Buritama, São Paulo; 27-II1996, H. brasiliensis, Barretos, São Paulo.

Observações. Dessa família foram coletadas somente deutoninfas hipopiais. Como várias de suas espécies são encontradas em ambientes bastante úmidos, como junto a exsudações de ferimentos de árvores (FLECHTMANN 1975), é possível que os hipopus encontrados nas folhas tenham se deslocado, para realizarem a forese, a partir do painel de sangria onde devem viver as outras fases de desenvolvimento ontogenético.

Até o momento, haviam sido registradas 11 espécies nominais de ácaros associados à seringueiras, pertencentes a 10 gêneros de quatro famílias. Três outras espécies foram identificadas somente ao nível genérico: Tydeus sp. (SILVA 1972), Lorryia sp. e Iphiseiodes sp. (FleChTMANN \& ARLeU 1984). Dessas espécies somente Allonychus brasiliensis (McGregor, 1950), registrada por SILVA (1972), não foi encontrada neste levantamento. 
O presente trabalho acrescenta nove espécies a essa lista (Typhlodromus annectens, Euseius citrifolius, E. concordis, Hemicheyletia wellsi, Lorryia formosa, L. boycei, Tydeus kenyensis, Atrichoproctus uncinatus e Tyrophagus putrescentiae), além de nove outras identificadas ao nível genérico (Daidalotarsonemus sp., Tarsonemus sp., Agistemus sp., Zetzellia sp., Pseudobonzia sp., Tydeus sp., Pronematus sp., Parapronematus sp. e Oulenzia sp.). Lorryia boycei e Tydeus kenyensis são registradas pela primeira vez no Brasil. O gênero Daidalotarsonemus DeLeon, 1956 é registrado pela primeira vez na América do Sul.

AGRADECIMENTOS. Aos Profs. Drs. Gilberto J. de Moraes e Carlos H.W. Flechtmann, Depto. de Zoologia, ESALQ-USP, Piracicaba, São Paulo, pela leitura crítica do manuscrito e valiosas sugestões apresentadas. À Profa. Dra. Marineide Rosa Vieira, Depto. de Biologia, FEIS-UNESP, Ilha Solteira, São Paulo e aos Engenheiros Agrônomos Anette Buuck Marques e Elivair Ferreira Marques, Fazenda Quimional Agropecuária Ltda., Planalto, São Paulo; Marcel R. Tanzini, Plantações E. Michelin, Rondonópolis, Mato Grosso; Vander Bassan Ruy, Fazenda Itaguaçu, Monte Aprazível, São Paulo, pelo envio de material para a realização destes estudos e fornecimento de informações técnicas a respeito de algumas das infestações observadas. Aos Engenheiros Agrônomos José Fernando Canuto Benesi, Diretor Técnico da Associação Paulista dos Produtores e Beneficiadores de Borracha (APABOR) e José Noronha Bacchiega, Delegacia Agrícola de São José do Rio Preto, São Paulo, pelo envio de material.

\section{REFERÊNCIAS BIBLIOGRÁFICAS}

Aranda, C.B.R. \& C.H.W. Flechtmann. 1971. A report on the Tetranychidae of Paraguay. Proc. Entomol. Soc. Wash. 73 (1): 29-33.

BAGDASARIAN, A.T. 1978. A new genus of eriophyid mites (Eriophyoidea). Zoologichesky Zhurnal (57): 936-939.

BAKER, E.W. 1944. Tideidos Mexicanos (Acari, Tydeidae). Rev. Soc. Mex. Hist. Natur. 5 (1-2): 73-81.

- 1945. Mites of the genus Tenuipalpus (Acarina: Trichadenidae). Proc. Entomol. Soc. Wash. 47 (2): 33-44.

-1949a. A review of the mites of the family Cheyletidae in the United States National Museum. Proc. U.S. Nat. Mus. 99 (3238): 267-320.

. 1949b. The genus Brevipalpus (Acarina: Pseudoleptidae). Am. Midl. Nat. 42 (2): 350-402. 1968. The genus Lorryia. Ann. Entomol. Soc. Amer. 61 (4): 986-1008.

1970. The genus Tydeus: Subgenera and species groups with descriptions of new species

(Acarina: Tydeidae). Ann. Entomol. Soc. Amer. 63 (1); 163-177.

BAKER, E.W. \& A.E. PRITCHARD. 1953. A review of the false spider mite genus Tenuipalpus Donnadieu

(Acarina: Phytoptipalpidae). Ann. Entomol. Soc. Amer. 46 (3): 317-336.

. 1960. The Tetranychoid mites of Africa. Hilgardia 29 (11): 455-574. 1962. Arañas rojas de America Central. Revta Soc. Hist. nat. 23: 309-340.

BAKER, E.W. \& D.M. TUTTLE. 1987. The false spider mites of Mexico (Tenuipalpidae: Acari). U.S. Department of Agriculture, Technical Bulletin (1706): 1-237.

BAKer, E.W. \& G.W. Wharton. 1952. An introduction to Acarology. New York, The MacMillan Company, 465p.

BAKER, E.W.; D.M. TUTtLE \& M.J. ABBATIELLO. 1975. The false spider mites of northwestern and north central Mexico (Acarina: Tenuipalpidae). Smithsn. Contrib. Zool. 194: 1-23.

BANKS, N. 1904. Class III, Arachnida, Order I, Acarina, four new species of injurious mites. Jour. N.Y. Entomol. Soc. 12: 53-56. 
BeER, R.E. \& A. Nucifora. 1965. Revisione dei generi della famiglia Tarsonemidae (Acarina). Boll. Zool. agric. Bachic., Ser. 2, 7: 19-43.

BondAR, G. 1928. Relatório. Bol. laborat. patologia vegetal, Bahia, 4: 39-46.

Chant, D.A. 1959. Phytoseiid mites (Acarina: Phytoseiidae). Part I. Bionomics of seven species in southeastern England. Part II. A taxonomic review of thefamily Phytoseiidae, with descriptions of thirty-eight new species. Canad. Entomol. 12 (Suppl.): 1-166p.

Chant, D.A. \& E.W. Baker. 1965. The Phytoseiidae (Acarina) of Central America. Mem. Entomol. Soc. Canada 41: 1-56.

CHANT, D.A. \& E. YoshidA-Shaul. 1984. A world review of the occidentalis species group in the genus Typhlodromus Scheuten (Acarina: Phytoseiidae). Canad. Jour. Zool. 62: 1860-1871.

Chiavegato, L.G. 1980. Ácaros da cultura dos citros, p. 469-501. In: O. RodRIGUEZ \& F.C.P. ViÉGAS (Ed.). Citricultura brasileira. Campinas, Fundação Cargill, 739p.

Comroy, H.L. 1958. A preliminary survey of the plant mites of Puerto Rico. Jour. Agric. Univ. Puerto Rico 42 (2): 39-144.

Cooreman, J. 1958. Notes et observations sur les Acariens. VII-Photia graeca n.sp. (Acaridia, Canestriniidae) et Lorryia formosa n.sp. (Stomatostigmata, Tydeydae). Bull. Inst. Roy. Sci. Nat. Belgique 34 (8): 1-10.

Deleon, D. 1958. Four new Typhlodromus from sothern Florida (Acarina: Phytoseiidae). Florida Entomol. 41: 73-76.

- 1961. The genus Brevipalpus in Mexico. Part II (Acarina: Tenuipalpidae). Florida Entomol. 44 (1): 41-52.

Denmark, H.A. \& M.H. Muma. 1970. Some Phytoseiid mites of Paraguay (Phytoseiidae: Acarina). Florida Entomol. 53 (4): 219-227.

Denmark, H.A. \& M.H. Muma. 1973. Phytoseiidae mites of Brazil (Acarina: Mesostigmata). Rev. Brasil. Biol. 33: 235-276.

EHARA, S. 1993. Two new species of the genus Agistemus Summers from Malayasia (Acari, Stigmaeidae). Jour. Acarol. Soc. Jpn. 2 (2): 79-82.

EwING, H.E. 1939. A revision of the mites of the subfamily Tarsoneminae of North America, the West Indies and the Hawaiian Islands. Tech. Bull. U.S. Dept. Agric. 653: 1-63.

Farias, A.R.; C.H.W. FlechtMAnN; G.J. DE Moraes \& J.A. MCMurTRY. 1981. Predadores do ácaro verde da mandioca, no nordeste do Brasil. Pesq. Agropec. Brasil. 16 (3): 313-317.

Fazolin, M. \& L.V. Pereira. 1989. Ocorrência de Oligonychus gossypii (Zacher 1920) (Acari: Tetranychidae) em seringueiras cultivadas. An. Soc. Entomol. Brasil 18 (1): 199-202.

FERES, R.J.F. 1992. A new species of Calacarus Keifer (Acari, Eriophyidae, Phyllocoptinae) from Hevea brasiliensis Muell. Arg. (Euphorbiaceae) from Brazil. Internat. Jour. Acarol. 18 (1): 61-65.

- 1998. Two new Phyllocoptine mites (Acari: Eriophyidae) from Hevea brasiliensis Muell. Arg. (Euphorbiaceae) from Brazil. Internat. Jour. Acarol. 24 (1): 69-74.

Feres, R.J.F. \& C.H.W. FleChtMAnN. 1995. Mites (Acari) associated with bamboo (Bambusa sp., Poaceae) in a wood area from northwestern São Paulo State, Brazil. Revta bras. Zool. 12 (3): 533-546.

Feres, R.J.F. \& G.J. DE Moraes. 1998. Phytoseiid mites (Acari: Phytoseiidae) from woody areas in the State of São Paulo, Brazil. Syst. Appl. Acarol. 3: 125-132.

FLeChTMANN, C.H.W. 1973. Lorryia formosa Cooremann, 1958 - Um ácaro dos citros pouco conhecido no Brasil. Ciência e Cultura 25 (12): 1179-1181.

- 1975. Elementos de Acarologia. São Paulo, Livraria Nobel S.A., 344p.

1976. A report on the Tetranychidae (Acari) of Brasil: an emmendation. Revta bras. Entomol. 20 (2): 115-116.

- 1979. Tuckerella ornata (Tucker), um ácaro novo para o Brasil e outros Tetranychoidea (Acari) do Estado do Pará. An. Esc. Sup. Agric. "Luiz de Queiroz", Piracicaba, 36: 615-620.

- 1986. Ácaros em produtos armazenados e na poeira domiciliar. Piracicaba, Fundação de Estudos Agrários Luiz de Queiroz-FEALQ, 97p. 
1989. Seringueira (Hevea sp.), um novo hospedeiro para Oligonychus gossypii (Zacher, 1920) (Acari: Tetranychidae). Bol. Mus. Para. Emílio Goeldi, sér. Zool., 5 (1): 127-128.

Flechtmann, C.H.W. \& J.M. Abreu. 1973. Ácaros fitófagos do Estado da Bahia, Brasil. Ciência e Cultura 25 (3): 244-251.

FLEChtMANN, C.H.W. \& R.J. ARLEU. 1984. Oligonychus coffeae (Nietner, 1861), um ácaro tetraniquídeo da seringueira (Hevea brasiliensis) novo para o Brasil e observações sobre outros ácaros desta planta. Ecossistema 9: 123-125.

Flechtmann, C.H.W. \& E.W. BAKER. 1970. A preliminary report on the Tetranychidae (Acarina) of Brazil. Ann. Entomol. Soc. Amer. 63 (1): 156-163.

. 1975. A report on the Tetranychidae (Acari) of Brazil. Revta. bras. Entomol. 19 (3): 111-122.

GEIJSKES, D.C. 1939. Beitraege zur Kenntnis der europeaischen Spinnmilben (Acari, Tetranychidae), mit besonderer Beruecksichtigung der niederlaendischen Arten. Meded. van de Landbouwhoogesch. te Wageningen (Nederland) 42 (4): 1-68.

Gonzalez, R.H. 1975. Revision of the Brevipalpus phoenicis "complex", with descriptions of new species from Chile and Thailand (Acarina, Tenuipalpidae). Acarologia 17 (1): 82-91.

GutIERREZ, J. 1968. Tetranychidae nouveaux de Madagascar (Quatrième note). Acarologia 10 (1): 13-28.

JePPSON, L.R.; H.H. KeIfER \& E.W. BAKER. 1975. Mites injurious to economic plants. Berkeley, University of California Press, XXIV+641p.

LINDQUisT, E.E. 1986. The world genera of Tarsonemidae (Acari: Heterostigmata): A morphological, Phylogenetic, and Systematic revision, with a reclassification of family-group taxa in the Heterostigmata. Mem. Entomol. Soc. Canada (136): 1-517.

McGregor, E.A. 1914. Four new tetranychids. Ann. Entomol. Soc. Amer. 7: 354-364.

. 1919. The red spiders of America and a few European species likely to be introduced. Proc. U.S. Nat. Mus. 56: 641-679.

. 1950. Mites of the family Tetranychidae. Amer. Midl. Nat. 44 (2): 257-420.

MEYER, M.K.P.S. 1974. A revision of the Tetranychidae of Africa (Acari) with a key to the genera of the world. Entomol. Mem. Dept. Agric. Tech. Serv. Repub. S. Afr. (36) 1-291.

- 1979. The Tenuipalpidae (Acari) of Africa with keys to the world fauna. Entomol. Mem. Dept. Agric. Tech. Serv. Repub. S. Afr. (50): 1-135.

1987. African Tetranychidae (Acari: Prostigmata), with reference to the world genera. Entomol. Mem. Dept. Agric. Wat. Supply Repub. S. Afr. (69): 1-175.

MEYER, M.K.P.S. \& M. DA C. RodRIGUES. 1965. Acari associated with cotton in Southern Africa (with reference to other plants). Garcia de Orta Lisboa 13 (2): 195-226.

Moraes, G.J. DE \& J.A. MCMurtry. 1983. Phytoseiid mites (Acarina) of Northeastern Brazil with descriptions of four new species. Internat. Jour. Acarol. 9: 131-148.

Moraes, G.J. DE \& J.V. OliveiRA. 1982. Phytoseiid mites of coastal Pernambuco, in northeastern Brazil. Acarologia 23: 315-318.

Moraes, G.J. DE; H.A. Denmark \& J.M. Guerrero. 1982. Phytoseiid mites of Colombia (Acarina, Phytoseiidae). Internat. Jour. Acarol. 8 (1): 15-22.

Moraes, G.J. DE; J.A. MCMUrTRY \& H.D. DenMARK. 1986. A Catalog of the mite family Phytoseiidae: references to taxonomy, synonymy, distribution and habitat. Brasilia, EMBRAPA-DDT, 353p.

MoraEs, G.J. DE \& N.C. MESA. 1988. Mites of the family Phytoseiidae (Acari) in Colombia, with descriptions of three new species. Internat. Jour. Acarol. 14 (2): 71-88.

Moraes, G.J. DE; N.C. MeSA \& A. BRAun. 1991. Some phytoseiid mites of Latin America (Acari: Phytoseiidae). Internat. Jour. Acarol. 17 (2): 177-139.

MoutiA, L.A. 1958. Contribution to the study of some phytophagous acarina and their predators in Mauritius. Bull. Entomol. Res. 49: 59-75.

Muma, M.H. 1961. Subfamiles, genera, and species of Phytoseiidae (Acarina: Mesostigmata). Florida St. Mus. Bull. Biol. Sci. 5: 267-302. 
1963. The genus Galendromus Muma, 1961 (Acarina: Phytoseiidae). Florida Entomol. Suppl. 1: 15-41.

. 1964. Cheyletidae (Acarina: Trombidiformes) associated with citrus in Florida. Florida Entomol. 47 (4): 239-253.

Muma, M.H.; H.A. Denmark \& D. Deleon. 1970. Phytoseiidae of Florida. Arthropods of Florida and nighboring land areas. Gainesville, Florida Dept. Agr. Cons. Serv., Div. Plant Ind., Vol. 6, $150 \mathrm{p}$.

NIETNER, J. 1861. Observations on the enemies of the coffee tree in Ceylon. 31p.

Oudemans, A.C. 1924. Acarologische Aanteekeningen. LXXIV. Entomol. Ber. Amst. 6: 249-260.

Paschoal, A.D. 1971. Nova relação de ácaros de plantas do Brasil. Revta Peru. Entomol. 14 (1): 174-176.

PRITCHARD, A.E. \& E.W. BAKER. 1955. A revision of the spider mites family Tetranychidae. San Francisco, Pacific Coast Entomological Society, Memoirs series, Vol. 2, 472p. 175-274.

RoDrigues, M. DA C. 1968. Acarina de Moçambique - Catálogo das espécies relacionadas com a agricultura. Agron. moçamb. 2 (4): 215-256.

SAYED, M.T. 1946. Description of Tenuipalpus granati nov. spec. and Brevipalpus pyri nov. spec. $1^{\text {er }}$ Ent. Bull. Soc. Fouad. $1^{\text {er }}$ 30: 99-104.

SCHRANK, F. VON DE P. 1781. "Enumeratio Insectorum Austriae Indiginorum, Austae Vindelicorum", p.507-524.

Silva, P. 1972. Pragas da seringueira no Brasil, problemas e perspectivas. An. $1^{\circ}$ Semin. Nac. Seringueira, Cuiabá, p.143-152.

Smiley, R.L. 1967. Some Tarsonemidae from the Republic of the Congo (Acarina). Proc. entomol. Soc. Wash. 66: 145-150.

. 1975. A generic revision of the mites of the family Cunaxidae (Acarina). Ann. Entomol. Soc. Amer. 68 (2): 227-244.

Summers, F.M. \& D.W. P. 1970. Revision of the family Cheyletidae. Univ. Calif. Publ. Entomol. 61: $1-153$.

TUCKER, R.W.E. 1926. Some South Africa mites. Mainly Tetranychidae and Eriophyidae. Union S. Africa Dept. Agr. Div. Ent. Mem. 5: 3-15.

Volgin, V.I. 1955. In: E.N. PAULovSKII. Acarina of rodents of the USSR fauna. Acad. Nauk. SSSR, Zool. Inst. Opredelitelipo Faune SSSR 59: 1-459.

Wood, T.G. 1967. New Zealand mites of the family Stigmaeidae (Acari, Prostigmata). Trans. Roy. Soc. N.Z., Zool., 9 (9): 93-139.

Woolley, T.A. 1987. Acarology: mites and human welfare. Fort Collins, Library of Congress cataloging in Publication, 484p.

ZACHER, F. 1921. Neue und wenig bekannte Spinnmilben. Ztschr. ang. Entomol. 7: 181-187.

ZuAlaGA, C. \& V. SALDARRIAGA. 1970. Reconocimiento, identificacion y algunas observaciones sobre dinamica de poblaciones de acaros, en citricos del valle del Cauca. Acta Agron. 20 (3/4): 114-141.

Recebido em 01.X.1998; aceito em 03.II. 2000. 